PROCEEDINGS OF THE

AMERICAN MATHEMATICAL SOCIETY

Volume 133, Number 7 , Pages 2137-2146

S 0002-9939(05)07799-3

Article electronically published on February 25, 2005

\title{
ON DECOMPOSITIONS OF BANACH SPACES OF CONTINUOUS FUNCTIONS ON MRÓWKA'S SPACES
}

\author{
PIOTR KOSZMIDER
}

(Communicated by Alan Dow)

\begin{abstract}
It is well known that if $K$ is infinite compact Hausdorff and scattered (i.e., with no perfect subsets), then the Banach space $C(K)$ of continuous functions on $K$ has complemented copies of $c_{0}$, i.e., $C(K) \sim c_{0} \oplus X \sim$ $c_{0} \oplus c_{0} \oplus X \sim c_{0} \oplus C(K)$. We address the question if this could be the only type of decompositions of $C(K) \nsim c_{0}$ into infinite-dimensional summands for $K$ infinite, scattered. Making a special set-theoretic assumption such as the continuum hypothesis or Martin's axiom we construct an example of Mrówka's space (i.e., obtained from an almost disjoint family of sets of positive integers) which answers positively the above question.
\end{abstract}

\section{INTRODUCTION}

This paper is concerned with a set-theoretic construction of a compact topological space which has interesting consequences in the isomorphic theory of Banach spaces, of continuous functions with the supremum norm. It was recently proved in 7 that there are Banach spaces $C(K)$ of continuous functions on compact connected $K$ with no non-trivial decomposition $C(K)=A \oplus B$. Actually it was only recently proved that there are at all such Banach spaces (not of continuous functions) 4]. In this paper we consider similar questions about the minimal possible space of operators or collection of projections or complemented subspaces in the context of $K$ compact and scattered.

Infinite scattered compact spaces have non-trivial convergent sequences of isolated points, which easily give rise to complemented copies of $c_{0}$ in the function space which implies that there are many operators including projections with ranges

Received by the editors July 24, 2003 and, in revised form, April 15, 2004.

2000 Mathematics Subject Classification. Primary 03E50, 46E15, 54G12.

Key words and phrases. Banach spaces of continuous functions, few operators, scattered spaces, almost disjoint families.

The author acknowledges support from CNPQ, Processo Número 300369/01-8, from FAPESP, Processo Número 02/03677-7 and from Centre de Recerca Matemática at Universidad Autonoma de Barcelona.

${ }^{1} \sim$ will denote an isomorphism of Banach spaces, i.e., a linear isomorphism which is continuous. $A$ is complemented in $X$ if and only if $X \sim A \oplus B$ for some $B$. This is equivalent to the existence of a projection $P: X \rightarrow A$, i.e., an onto bounded operator such that $P^{2}=P([17]$, $\S 21)$. Since finite-dimensional or finite-co-dimensional subspaces are always complemented, by nontrivial decompositions we mean those with two infinite-dimensional factors (see [17, [11]). Unless stated otherwise all compact spaces are infinite and Hausdorff and all Banach spaces are infinite-dimensional. 
included in $c_{0}$. We ask in this paper if it is possible that there are no more operators and no more complemented subspaces in a $C(K) \nsim c_{0}$ for a scattered $K$. In section 2 , we obtain consistent examples.

Theorem. Assuming CH or MA there is a compact separable scattered Hausdorff space with $C(K) \nsim c_{0}$ such that all operators $T: C(K) \rightarrow C(K)$ are of the form $c I+S$ where $c$ is a real and $S$ has range included in a subspace isomorphic to $c_{0}$. Moreover the only decompositions $C(K)=A \oplus B$ into two infinite-dimensional complemented subspaces are such that $A \sim c_{0}$ and $B \sim C(K)$ or $A \sim C(K)$ and $B \sim c_{0}$.

Note that such a space $K$ must be non-metrizable since for separable $C(K)$ 's we have that the space is isomorphic to its square and that operators into $c_{0}$ have relatively simple structure, i.e., they correspond to weakly* null sequences in the dual to $C(K)$. Note that the question of the existence of Banach spaces whose only operators are scalar multiples of the identity plus an operator with separable range has been considered by various authors [18], 19], 20, [6]. We do not know if any set-theoretic assumption is needed for our $C(K)$ or even to get an example of any Banach space having the above properties, i.e., $c_{0} \nsim X \sim c_{0} \oplus X$ and whether this type of a non-trivial decomposition is the only one. We know, however, that the existence of $C(K) \nsim c_{0}$ which is Lindelö in the weak topology and has the above properties is consistent and independent from the axioms of ZFC. This result will be published elsewhere.

In this paper we only analyze the Banach space of continuous functions on Mrówka's or Isbell's space, also known as the $\Psi$-space, which was first considered by Alexandroff. If $\mathcal{A}=\left\{A_{\xi}: \xi<\kappa\right\}$ is an infinite almost disjoint (i.e., with finite pairwise intersections) family of subsets of $N$, then the space $K_{\mathcal{A}}$ is the Stone space of the Boolean algebra generated by $\mathcal{A}$. The Banach space of continuous functions on $K_{\mathcal{A}}$ was considered for example in [5] (example 2) or in [12] (example 8). There are three types of ultrafilters-points of $K_{\mathcal{A}}$ : first the isolated points corresponding to principal ultrafilters generated by singletons of $n \in N$; the second level is made up from ultrafilters generated by $A_{\xi}$ and the cofinite sets (these points will be denoted $x_{\xi}$ ); the third level is represented by the unique ultrafilter which contains all cofinite sets and the complements of $A_{\xi}$ 's (this point will be denoted by $\infty)$. We will use the same notation $A_{\xi}$ for the subset of $N$ and the corresponding clopen subset of $K_{\mathcal{A}}$ hoping that the meaning is clear from the context. The sets $A_{\xi}-\{0, \ldots, n-1\}$ will be denoted by $A_{\xi}^{n}$, the characteristic functions of $n \in N$ will be denoted by $e_{n} \in C\left(K_{\mathcal{A}}\right)$ and the characteristic functions of the $A_{\xi}^{n} \cup\left\{x_{\xi}\right\}$ 's will be denoted by $d_{\xi}^{n} \in C\left(K_{\mathcal{A}}\right)$. $C_{0}\left(K_{\mathcal{A}}\right)$ denotes the space of functions which vanish at $\infty$. The following are two natural questions, which appear in the light of the results of this paper:

Question 1. Is there a separable Banach space $X \nsim c_{0}$ whose only non-trivial decompositions are of the form $c_{0} \oplus X$ ?

Question 2. Are there (with no additional set-theoretic assumption) almost disjoint families such that the only decompositions of $C\left(K_{\mathcal{A}}\right)$ into two factors have one factor separable?

Let us make more comments on the second question. One can prove (Lemma 4) that the property of having few decompositions in the sense of our theorem for a 
$C(K)$ where $K$ is Mrówka's space is equivalent to the fact that the only decompositions of $C(K)$ have just one separable factor. Easy examples of decompositions for $A \subseteq N$ are of the form

$$
C(K) \sim C_{0}(\bar{A}) \oplus C_{0}(\overline{N-A}) \oplus R,
$$

where for every $A_{\xi} \in \mathcal{A}$ one has either $A_{\xi}-A$ is finite or $A \cap A_{\xi}$ is finite. Such an $A$ is called a separation of $\mathcal{A}$. The projection on the first factor is given by $P_{1}(f)=(f-f(\infty)) \mid \bar{A}$, and similarly for the second factor, while the last projection is given by $P_{3}(f)=f(\infty)$.

Thus the family $\mathcal{A}$ as in the theorem cannot have separations into two uncountable parts. There are two known examples of almost disjoint families with this property which, unlike our theorem, do not require any additional set-theoretic assumptions. One, due to Lusin ([10], [3], 4.1), is of cardinality $\omega_{1}$, and the other due to Mrówka [13, 3.11] is maximal and of cardinality $2^{\omega}$.

Section 3 is devoted to showing that Lusin's family cannot witness the theorem in ZFC. It turns out that under Martin's axiom all spaces $C\left(K_{\mathcal{A}}\right)$ for $\mathcal{A}$ of cardinality less than $2^{\omega}$ have many decompositions into both non-separable factors (even though, as in the case of Lusin's family, there may be no separations of the family into two uncountable parts). On the other hand, Mrówka's families could serve to prove the existence of spaces such as in the theorem without the use of any special set-theoretic assumptions. As Mrówka's argument relies on the perfect set property of Borel sets of the reals (we thank A. Miller for explaining to us this construction in [11), it is reasonable to expect that the positive answer could be obtained by the methods of descriptive set-theory. In the light of our result from section 3 it is natural to ask the following:

Question 3. Suppose MA. Is it true that if $|\mathcal{A}|=\left|\mathcal{A}^{\prime}\right|<2^{\omega}$, then $C\left(K_{\mathcal{A}}\right)$ is isomorphic to $C\left(K_{\mathcal{A}^{\prime}}\right)$ ?

In the context of the Pełczyński decomposition method ([14], 11], Appendix) one could ask:

Question 4. Suppose MA. Is it true that if $|\mathcal{A}|<2^{\omega}$, then $C\left(K_{\mathcal{A}}\right)$ is isomorphic to its square?

It seems also that it is unknown 2 in ZFC (of course our result decides it under a special set-theoretic assumption):

Question 5. Are there two almost disjoint families $\mathcal{A}$ and $\mathcal{A}^{\prime}$ of the same cardinality such that $C\left(K_{\mathcal{A}}\right)$ is not isomorphic to $C\left(K_{\mathcal{A}^{\prime}}\right)$ ?

Our terminology follows [17, [3], 2]. In particular we consider several interrelated structures such as Boolean algebras, compact spaces, Banach spaces of continuous functions, Radon measures, etc. The main links between them are given by the Stone duality and the Riesz representation theorem. We also use Rosenthal's lemma ([16, 2]), which can be formulated in our context as follows: given a sequence of absolutely convergent series $\sum_{n=0}^{\infty} m_{n}^{k}$ whose sums are bounded by the same number and given an $\varepsilon>0$, one can find an infinite set $M \subseteq N$ such that $\sum_{n \in M-\{k\}} m_{n}^{k}<\varepsilon$ for every $k \in M$.

\footnotetext{
${ }^{2}$ Mrówka shows in [13], 3.5, with no additional set-theoretic assumption, that there are $2^{2^{\omega}}$ non-homeomorphic spaces $K_{\mathcal{A}}$, and it follows from [13], 3.6 that there is one $K_{\mathcal{A}}$ such that $C\left(K_{\mathcal{A}}\right)$ has $2^{\omega}$ decompositions into nonseparable factors.
} 
Section 3, which deals with Martin's axiom, uses the terminology of chapter 2 of 8]. The terminology regarding the combinatorics of sets of integers follows [3]; in particular, $A \subseteq \subseteq^{*} B$ means that $A-B$ is finite, and almost disjoint means with finite intersection. All almost disjoint families are assumed to be infinite and consist of infinite sets. A pseudointersection of a family of sets $\mathcal{F}$ is an infinite set $A$ such that $A \subseteq^{*} B$ for all $B \in \mathcal{F}$. The number $p$ is the minimal cardinal such that there is a centred (intersections of finite subfamilies are infinite) family of sets of integers of cardinality $p$ without a pseudointersection. Martin's axiom (and so the continuum hypothesis) implies $p=2^{\omega}$. An ideal of subsets of $N$ is a family of proper subsets of $N$ which contains all finite sets and is closed under subsets and finite unions.

\section{Spaces of Continuous functions on MrówKa's SPACES WITH FEW OPERATORS}

Lemma 1. Assume that $p=2^{\omega}$. Suppose that $\mathcal{I}$ is an ideal of subsets of $N$ which is generated by $<2^{\omega}$ sets. Suppose that $\mathcal{F}$ is a family of finite subsets of $N$ such that for every $I \in \mathcal{I}$ there is an $F \in \mathcal{F}$ such that $I \cap F=\emptyset$. Then there is a sequence $\left(F_{n}\right)_{n \in N}$ of pairwise disjoint elements of $\mathcal{F}$ such that $I \cap \bigcup\left\{F_{n}: n \in N\right\}$ is finite for all $I \in \mathcal{I}$.

Proof. The assumption $p=2^{\omega}$ says that any centred family of subsets of $N$ of cardinality $<2^{\omega}$ has a pseudointersection (see [3]), i.e., a set which is almost included in all members of the family. Identify $N$ with the family $[N]^{<\omega}$ of all finite subsets of $N$ and consider a centred family

$$
\left\{[N-I]^{<\omega} \cap \mathcal{F}: I \in \mathcal{I}\right\} .
$$

Let $\mathcal{G}$ be its pseudointersection. It exists since $\mathcal{I}$ is generated by less than $p$ sets. Any infinite subset of $\mathcal{G}$ is another pseudointersection. So, using the fact that ideals contain all finite sets of $N$, one can choose a pairwise disjoint sequence which is a pseudointersection as well. It satisfies the lemma.

Proposition 2. Assume that $p=2^{\omega}$. There is an almost disjoint family $\mathcal{A}$ such that every bounded linear operator on $C_{0}\left(K_{\mathcal{A}}\right)$ is of the form $c I+S$ where $c$ is a constant and $S$ has separable range.

Proof. Before the construction, fix an enumeration $\left(m_{n}^{k}(\beta): n, k \in N, \beta<2^{\omega}\right)$, with cofinally many repetitions, of all real sequences $\left(m_{n}^{k}: n, k \in N\right)$ such that $\sum_{n=0}^{\infty}\left|m_{n}^{k}\right|<\rho$ for any $k \in N$ and some $\rho>0$. Construct the almost disjoint family from three parts:

$$
\left(A_{\beta}: \beta<2^{\omega}\right)=\left(B_{\beta}: \beta<2^{\omega}\right) \cup\left(C_{\beta}: \beta<2^{\omega}\right) \cup\left(D_{\beta}: \beta<2^{\omega}\right)
$$

by induction in $\beta<2^{\omega}$. Let $\mathcal{I}_{\beta}$ denote the ideal generated by $\left(B_{\alpha}, C_{\alpha}, D_{\alpha}: \alpha<\beta\right)$ and the finite subsets of $N$. Suppose that $B_{\alpha}, C_{\alpha}, D_{\alpha}$ for $\alpha<\beta$ are already constructed. At the stage $\beta$ we inquire if the following is satisfied for some fixed $\varepsilon>0$ :

$$
\forall A \in \mathcal{I}_{\beta} \exists F \in[N-A]^{<\omega} \exists k \in N-(A \cup F)\left|\sum_{n \in F} m_{n}^{k}(\beta)\right|>\varepsilon .
$$

If 1 ) is not satisfied, then $B_{\beta}$ and $C_{\beta}$ are arbitrary as long as they are almost disjoint and almost disjoint from the sets already constructed; the assumption $p=2^{\omega}$ implies that we can always find such sets since $p=2^{\omega}>\beta(p \leq a$ in [3] $)$. 
If 1) is satisfied, then $B_{\beta}=\bigcup\left\{F_{i}: i \in 2 N\right\}$ and $C_{\beta}=\left\{k_{i}: i \in N\right\}$ where the $F_{i}$ 's are pairwise disjoint finite sets and the $k_{i}$ 's are distinct positive integers, such that $k_{i} \notin F_{j}$ for $i, j \in N$ and

$$
\begin{gathered}
\forall i \in N \quad\left|\sum_{n \in F_{i}} m_{n}^{k_{i}}(\beta)\right|>\varepsilon, \\
\forall i \in N \sum_{j \neq i} \sum_{n \in F_{j}}\left|m_{n}^{k_{i}}(\beta)\right|<\varepsilon / 3 .
\end{gathered}
$$

It follows from Lemma 1 applied to the $F \cup\{k\}$ 's such as in 1) and $\mathcal{I}_{\beta}$ that there exist $F_{n}$ 's and $k_{n}$ 's satisfying 2 ) such that the corresponding $B_{\beta}, C_{\beta}$ are almost disjoint from the previously constructed sets $B_{\alpha}, C_{\beta}$ and $D_{\alpha}$ for $\beta<2^{\omega}$. Rosenthal's lemma implies that one can thin out the sequence obtaining 3 ).

Define $\mathcal{J}_{\beta}$ to be the ideal generated by the sets of $\mathcal{I}_{\beta}$ and $B_{\beta}, C_{\beta}$. Before the construction of $D_{\beta}$ we inquire if the following is satisfied for some fixed reals $r_{1}<r_{2}$ :

$$
\forall A \in \mathcal{J}_{\beta} \exists k, l \in N-A m_{k}^{k}<r_{1}<r_{2}<m_{l}^{l} .
$$

If 4 ) is not satisfied, then $D_{\beta}$ is arbitrary as long as it is almost disjoint from the sets already constructed. Again, it exists, since $p=2^{\omega}>\beta$.

If 4) is satisfied, then pick some $r_{1}<r_{2}$ witnessing it and define $D_{\beta}=\left\{k_{n}: n \in\right.$ $N\} \cup\left\{l_{n}: n \in N\right\}$ where $k_{n} \neq l_{j}$ for all $n, j \in N$ and

$$
\begin{gathered}
\forall n \in N \quad m_{k_{n}}^{k_{n}}<r_{1}<r_{2}<m_{l_{n}}^{l_{n}}, \\
\forall n \in N \quad \sum_{j \neq n}\left|m_{l_{j}}^{l_{n}}\right|<\frac{r_{2}-r_{1}}{6}, \sum_{j \neq n}\left|m_{k_{j}}^{k_{n}}\right|<\frac{r_{2}-r_{1}}{6}, \\
\forall n \in N \quad \sum_{j \in N}\left|m_{k_{j}}^{l_{n}}\right|<\frac{r_{2}-r_{1}}{6}, \quad \sum_{j \in N}\left|m_{l_{j}}^{k_{n}}\right|<\frac{r_{2}-r_{1}}{6} .
\end{gathered}
$$

Let us justify the existence of such a $D_{\beta}$. It follows from Lemma 1 applied to $\{k, l\}$ satisfying 4) that there exist two infinite disjoint sets $K, L \subseteq N$ such that $m_{k}^{k}<r_{1}<r_{2}<m_{l}^{l}$ for every $k \in K$ and $l \in L$ and both $K$ and $L$ are almost disjoint from the previously constructed sets $B_{\alpha}, C_{\alpha}$ for $\alpha<\beta$ and from $B_{\beta}, C_{\beta}$. Thus, any enumeration of $K=\left\{k_{n}^{\prime}: n \in N\right\}$ and $L=\left\{l_{n}^{\prime}: n \in N\right\}$ would give 5). To obtain 6 ) and 7 ) one needs to apply Rosenthal's lemma to $\frac{r_{2}-r_{1}}{6}$ and the series

$$
\sum_{i=0}^{\infty}\left(\left|m_{k_{i}^{\prime}}^{k_{n}^{\prime}}\right|+\left|m_{l_{i}^{\prime}}^{k_{n}^{\prime}}\right|+\left|m_{k_{i}^{\prime}}^{l_{n}^{\prime}}\right|+\left|m_{l_{i}^{\prime}}^{l_{n}^{\prime}}\right|\right)
$$

for all $n \in N$ obtaining infinite $M \subseteq N$ as in Rosenthal's lemma (see the introduction). Now consider two disjoint infinite sets $M_{1}, M_{2} \subseteq M$ and enumerate $\left\{k_{n}: n \in N\right\}=M_{1}$ and $\left\{l_{n}: n \in N\right\}=M_{2}$. 6) and 7) are satisfied by the construction. This completes the construction.

Let $T: C_{0}\left(K_{\mathcal{A}}\right) \rightarrow C_{0}\left(K_{\mathcal{A}}\right)$. Define $g(n)=T\left(e_{n}\right)(n)$. Define $m_{n}^{k}=T\left(e_{n}\right)(k)$, i.e., $g(n)=m_{n}^{n}$.

Claim 1. There is a countable set $X \subseteq 2^{\omega}$ such that for every $\beta \in 2^{\omega}-X$, for every $n, k \in N$ we have

$$
T\left(d_{\beta}^{n}\right)(k)=\sum_{i \in A_{\beta}^{n}} T\left(e_{i}\right)(k) .
$$


Proof of the Claim. Note that $\phi_{k}(f)=T(f)(k)=T^{*}\left(\delta_{k}\right)$ for $k \in N$ is a bounded linear functional on $C_{0}\left(K_{\mathcal{A}}\right)$. Thus by the Riesz representation theorem (see [17, 18.5.3) there is a Radon measure $\mu_{k}$ on $K_{\mathcal{A}}$ such that $\mu_{k}(\{\infty\})=0$ which satisfies $T(f)(k)=\int f d \mu_{k}$ for all $f \in C\left(K_{\mathcal{A}}\right)$. Since $K_{\mathcal{A}}$ is scattered, all Radon measures on $K_{\mathcal{A}}$ are atomic (see [17, 19.7.6); in particular, they have countable carriers. Let $X$ be a countable set which includes the intersections of all the carriers of the $\mu_{k}$ 's with the $\left\{x_{\beta}: \beta<2^{\omega}\right\}$. If $\beta \notin X$, then $\mu_{k}\left(\left\{x_{\beta}\right\}\right)=0$ for all $k \in N$ and so

$$
T\left(d_{\beta}^{n}\right)(k)=\int_{K_{A}-\left\{x_{\beta}\right\}}\left[\sum_{i \in A_{\beta}^{n}} e_{i}+\chi_{\left\{x_{\beta}\right\}}\right] d \mu_{k}=\sum_{i \in A_{\beta}^{n}} T\left(e_{i}\right)(k),
$$

since $\chi_{\left\{x_{\beta}\right\}}$ is Borel and so integrable with respect to any Radon measure, as required.

Before proceeding to Claim 2, note that $m_{n}^{k}=\mu_{k}(\{n\})$. This implies that $\sum_{n \in N}\left|m_{n}^{k}\right| \leq\left\|\mu_{k}\right\| \leq\|T\|$ for all $k \in N$. It follows that the sequence of the series $\left(m_{n}^{k}: n, k \in N\right)$ appears for cofinally many $\beta<2^{\omega}$ on our list $\left(m_{n}^{k}(\beta): n, m \in\right.$ $\left.N, \beta<2^{\omega}\right)$.

Claim 2. There is a constant $c$ and a countable set $Y \subseteq 2^{\omega}$ such that for every $\beta \in 2^{\omega}-Y$ we have

$$
\lim _{n \in A_{\beta}} g(n)=c .
$$

Proof of the Claim. Suppose that the claim is false, i.e., for every countable $Y \subseteq 2^{\omega}$ there are $\alpha, \beta \in 2^{\omega}-Y$ and two rationals $q_{1}<q_{2}$ such that

$$
\liminf _{n \in A_{\alpha}} g(n)<q_{1}^{\alpha, \beta}<q_{2}^{\alpha, \beta}<\limsup _{n \in A_{\beta}} g(n) .
$$

Knowing that every element of the ideal is included in the union of finitely many generators, without loss of generality we may assume that there are two reals $r_{1}<r_{2}$ such that

$$
\forall A \in \mathcal{I}_{2 \omega} \exists k, l \in N-A \quad g(k)<r_{1}<r_{2}<g(l) .
$$

But $g(k)=m_{k}^{k}(\beta), g(l)=m_{l}^{l}(\beta)$ for some $\beta<2^{\omega}$, i.e., 4) is satisfied. As our initial enumeration has cofinally many repetitions we may assume that $\beta \notin X$ from Claim 1. Now Claim 1, 5),6) and 7) imply that

$$
\begin{aligned}
& T\left(\chi_{D_{\beta}}\right)\left(k_{n}\right)<r_{1}+\frac{r_{2}-r_{1}}{3}, \\
& T\left(\chi_{D_{\beta}}\right)\left(l_{n}\right)>r_{2}-\frac{r_{2}-r_{1}}{3},
\end{aligned}
$$

which means that $T\left(\chi_{D_{\beta}}\right)$ is discontinuous at the point of $K_{\mathcal{A}}$ corresponding to $D_{\beta}$, a contradiction.

Claim 3. $T-c I$ has separable range.

Proof of the Claim. Suppose the claim is false. Then there is an $\varepsilon>0$ such that for every countable $Z \subseteq 2^{\omega}$ and every $l^{\prime} \in N$ there are $\alpha, \beta \in 2^{\omega}-Z$ and $l \geq l^{\prime}$ such that $\left|(T-c I)\left(d_{\beta}^{l}\right)\left(x_{\alpha}\right)\right|>\varepsilon$. By the continuity of the functions $(T-c I)\left(d_{\beta}^{l}\right)$, this means that $\left|(T-c I)\left(d_{\beta}^{l}\right)(k)\right|>\varepsilon$ for $k \in A_{\alpha}$ for sufficiently large $k$. Let $X$ be 
as in Claim 1 and suppose that $X \subseteq Z$. Let $Y$ be as in Claim 2 and suppose that $Y \subseteq Z$. Claims 1 and 2 imply that

$$
\left|T\left(d_{\beta}^{l}\right)(k)-c d_{\beta}^{l}(k)\right|=\left|\sum_{n \in A_{\beta}^{l}}\left[T\left(e_{n}\right)(k)-c d_{\beta}^{l} e_{k}\right]\right|=\left|\sum_{n \in A_{\beta}^{l}-\{k\}} m_{n}^{k}\right|>\varepsilon
$$

if $k$ is sufficiently large in $A_{\alpha}$, since by Claim 2, then $c$ is close to $g(k)=T\left(e_{k}\right)(k)=$ $m_{k}^{k}$ and $d_{\beta}^{l}(k)=0$ if $k \notin A_{\beta}^{l}$ and $d_{\beta}^{l}(k)=1$ if $k \in A_{\beta}^{l}$ (note that we need to consider the case of $k \in A_{\beta}$ only because it could happen that $\alpha=\beta$ ). It follows that 1 ) is satisfied at stage $\beta$ such that $m_{n}^{k}=m_{n}^{k}(\beta)$ for all $n, k \in N$. Since our enumeration has cofinally many repetitions and $c f\left(2^{\omega}\right)>\omega$ we may assume that $\beta \notin X, Y$ where $X$ is as in Claim 1 and $Y$ is as in Claim 2.

Thus 2) and 3) is satisfied for $B_{\beta}$ and $C_{\beta}$. But this implies that $\left|T\left(\chi_{B_{\beta}}\right)\left(k_{i}\right)\right|>$ $2 \varepsilon / 3$ if $i$ is even and $\left|T\left(\chi_{B_{\beta}}\right)\left(k_{i}\right)\right|<\varepsilon / 3$ if $i$ is odd, which contradicts the fact that $T\left(\chi_{B_{\beta}}\right)$ must be continuous at the point corresponding to $C_{\beta}$. This completes the proof of the proposition.

Lemma 3 (5], Example 2c). Suppose that $A \subseteq C_{0}\left(K_{\mathcal{A}}\right)$ is separable for some almost disjoint $\mathcal{A}$. Then there is a closed subspace $B$ such that $A \subseteq B$ and $B \sim c_{0}$.

Lemma 4. Suppose that $C_{0}\left(K_{\mathcal{A}}\right)=A \oplus B$ where $A$ is separable and $B$ is not separable. Then $B$ has a complemented copy of $c_{0}$. In particular $A \sim c_{0}$ and $B \sim C_{0}\left(K_{\mathcal{A}}\right)$.

Proof. By Lemma 3, $A$ is complemented in an isomorphic copy of $c_{0}$; thus by a result of 14] (Theorem 1), $A \sim c_{0}$. Well order $\mathcal{A}$ as $\left(A_{\xi}: \xi<\kappa\right)$ for some uncountable cardinal $\kappa$ so that $A$ is included in the closure of $\left\{\chi_{A_{n}}: n \in \omega\right\} \cup\left\{e_{n}: n \in N\right\}$. Let $P$ be the projection on $B$ and $Q$ the projection on $A$. By induction construct $\omega<\beta_{i}<\beta_{i}^{\prime}<\beta_{i+1}<\ldots<\omega_{1}$ and $n_{i} \in N$ such that

$$
\begin{gathered}
\left\|Q\left(d_{\beta_{i}}^{n_{i}}\right)-Q\left(d_{\beta_{i}^{\prime}}^{n_{i}}\right)\right\| \leq 1 / 2^{i}, \\
\left(A_{\beta_{i}}^{n_{i}} \cup A_{\beta_{i}^{\prime}}^{n_{i}}\right) \cap\left(A_{\beta_{j}}^{n_{j}} \cup A_{\beta_{j}^{\prime}}^{n_{j}}\right)=\emptyset
\end{gathered}
$$

for all $i<j<\omega$. This can be accomplished since $\left(A_{\xi}: \xi<\kappa\right)$ is almost disjoint and $A$ is separable. We claim that

$$
X=\left\{\sum_{i \in N} a_{i} P\left(d_{\beta_{i}}^{n_{i}}-d_{\beta_{i}^{\prime}}^{n_{i}}\right):\left(a_{i}\right)_{i \in N} \in c_{0}\right\}
$$

is included in $B$, that it is isomorphic to $c_{0}$ and that it is complemented in $C_{0}\left(K_{\mathcal{A}}\right)$ and hence in $B$.

First note that each element of $X$ is a well-defined element of $C_{0}\left(K_{\mathcal{A}}\right)$ because the series $\sum_{i \in N} a_{i} P\left(d_{\beta_{i}}^{n_{i}}-d_{\beta_{i}^{\prime}}^{n_{i}}\right)$ is norm convergent. For this we use the fact that $P+Q$ is the identity to write

$$
P\left(d_{\beta_{i}}^{n_{i}}-d_{\beta_{i}^{\prime}}^{n_{i}}\right)=\left(d_{\beta_{i}}^{n_{i}}-d_{\beta_{i}^{\prime}}^{n_{i}}\right)-\left[Q\left(d_{\beta_{i}}^{n_{i}}\right)-Q\left(d_{\beta_{i}^{\prime}}^{n_{i}}\right)\right]
$$

and we note that $\sum_{i \in N} a_{i}\left(d_{\beta_{i}}^{n_{i}}-d_{\beta_{i}^{\prime}}^{n_{i}}\right)$ norm converges by 9$)$ and $\sum_{i \in N} a_{i} Q\left(d_{\beta_{i}}^{n_{i}}-d_{\beta_{i}^{\prime}}^{n_{i}}\right)$ norm converges by 8$). X$ is included in $B$ because $B$ is closed. The projection on $X$ is

$$
R(f)=\sum_{i \in N} f\left(x_{\beta_{i}}\right) \cdot P\left(d_{\beta_{i}}^{n_{i}}-d_{\beta_{i}}^{k_{i}}\right) .
$$


It is linear and bounded and hence continuous by 10). By the fact that all elements of the space $A$ are zero on $x_{\beta_{i}}$ (since $\beta_{i}>\omega$ ), $R$ is zero on $A$, and so it is true that

$$
R\left(P\left(d_{\beta_{i}}^{n_{i}}-d_{\beta_{i}}^{n_{i}}\right)\right)=R\left(d_{\beta_{i}}^{n_{i}}-d_{\beta_{i}}^{n_{i}}\right)=P\left(d_{\beta_{i}}^{n_{i}}-d_{\beta_{i}}^{n_{i}}\right),
$$

i.e., $R$ is the identity on $X$. To prove the last part of the lemma let $B \sim c_{0} \oplus C$ (since $B$ by the above has a complemented copy of $c_{0}$ ) and note that

$$
C_{0}\left(K_{\mathcal{A}}\right) \sim A \oplus B \sim c_{0} \oplus c_{0} \oplus C \sim c_{0} \oplus C \sim B
$$

Proof of the Theorem. Let $\mathcal{A}$ be as in Proposition 2. Since every operator on $C\left(K_{\mathcal{A}}\right)$ is an operator on $C_{0}\left(K_{\mathcal{A}}\right)$ plus an operator with finite-dimensional range (restriction projected on $C_{0}\left(K_{\mathcal{A}}\right)$ by subtracting the value at $\infty$ ), the operators on $C\left(K_{\mathcal{A}}\right)$ are of the desired form by Proposition 2.

Suppose that $P$ is any projection on $C\left(K_{\mathcal{A}}\right)$, in particular $P=P^{2}$, so if $P=$ $c I+S$ where $S$ has a separable range, we get $c I+S=c^{2} I+2 c S+S^{2}$, that is, $\left(c-c^{2}\right) I$ has separable range, which means that $c=c^{2}$. If $c=0, P$ has separable range; otherwise the projection on the complement has separable range since the sum of the projections is $I$. The conclusion is that the only decompositions of $C\left(K_{\mathcal{A}}\right)$ are into one separable and one nonseparable summand. Lemma 4 implies that the separable summand is a copy of $c_{0}$ and the nonseparable summand is a copy of $C\left(K_{\mathcal{A}}\right)$ as required.

\section{Decompositions of $C\left(K_{\mathcal{A}}\right)$ for small $\mathcal{A}$ under Martin's axiom}

Lemma 5. Suppose that $\kappa$ is an infinite cardinal. Assume $M A_{\kappa}$ and suppose that an almost disjoint family $\mathcal{A}=\left\{A_{\xi}: \xi<\kappa\right\}$ is of cardinality $\kappa$. For every $X \subseteq \kappa$ there is a finite-to-one function $f: N \rightarrow N$ such that $f \circ f=f$ and 1 ) $\left(f\left[A_{\xi}\right] \cup f^{-1}\left[A_{\xi}\right]\right) \subseteq{ }^{*} A_{\xi}$ for all $\xi \in X$, 2) $f[N] \cap A_{\eta}$ is finite for all $\eta \in \kappa-X$.

Proof. First we define a partial order $P$ to which we will be applying Martin's axiom. The conditions of $P$ are of the form $p=\left(a_{p}, n_{p}, f_{p}\right)$, where $a_{p} \in[\kappa]^{<\omega}$, $n_{p} \in N, f_{p}: n_{p} \rightarrow n_{p}$ such that $f_{p} \circ f_{p}=f_{p}$ and

a) for all distinct $\xi, \eta \in a_{p}$ we have $A_{\xi} \cap A_{\eta} \subseteq n_{p}$. The order on $P$ is defined by $p \leq q$ if and only if $a_{p} \supseteq a_{q}, n_{p} \geq n_{q}, f_{p} \supseteq f_{q}$ and

b) $f_{p}^{-1}(\{n\})=f_{q}^{-1}(\{n\})$ for all $n<n_{q}$,

c) for $\xi \in a_{q} \cap X$ we have $n \in A_{\xi} \cap\left[n_{q}, n_{p}\right)$ iff $f(n) \in A_{\xi} \cap\left[n_{q}, n_{p}\right)$,

d) $f\left[\left[n_{q}, n_{p}\right)\right] \cap A_{\eta}=\emptyset$ for $\eta \in a_{q}-X$.

Note that the above relation is a partial order. To be able to take advantage of Martin's axiom, we must prove that $P$ satisfies the countable chain condition. Suppose that an uncountable family of conditions of $P$ is given. We may assume without loss of generality that their second coordinates are all equal to some integer $n$ and that all third coordinates are equal to some $f: n \rightarrow n$. So it is enough to prove that any two conditions $q, r$ with $n=n_{q}=n_{r}$ and $f=f_{q}=f_{r}$ are compatible.

Find $n_{p}>n$ so that for all $\xi \in\left(a_{q} \cup a_{r}\right) \cap X$ there is an $n_{\xi} \in\left[n, n_{p}\right)$ which does not belong to any $A_{\eta}$ for $\eta$ distinct from $\xi$ and $\eta \in a_{q} \cup a_{r}$; moreover, $\left[n, n_{p}\right.$ ) should contain an $m$ which does not belong to any of the sets $A_{\xi}$ for $\xi \in a_{q} \cup a_{r}$, and we also require that $A_{\xi} \cap A_{\eta} \subseteq n_{p}$ for distinct $\xi, \eta \in a_{q} \cup a_{r}$. This can be accomplished because the sets are almost disjoint. Put $a_{p}=a_{q} \cup a_{r}$. For $k \in n$ put $f_{p}(k)=f_{q}(k)=f_{r}(k)$ and for $k \in\left[n, n_{p}\right)$ define $f_{p}(k)$ as follows:

i) if $k \in A_{\xi} \cap A_{\xi^{\prime}}$ for distinct $\xi, \xi^{\prime} \in\left(a_{q} \cup a_{r}\right) \cap X$, then $f_{p}(k)=k$; 
ii) if $n \in A_{\xi}$ for $\xi \in\left(a_{q} \cup a_{r}\right) \cap X$, but i) doesn't hold, then $f(k)=n_{\xi}$;

iii) if neither i) nor ii) holds for $k$, then $f(k)=m$.

It is clear that $p=\left(a_{p}, n, f\right)$ is a condition of $P$. Let us check that $p \leq q, r$. Conditions b) and c) are clear from the construction.

The key observation needed to prove d) is that if i) holds for $k$, then $\xi \in a_{q}-a_{r}$ and $\xi^{\prime} \in a_{r}-a_{q}$. This is because condition a) for $q$ or $r$ implies that the intersections $A_{\xi} \cap A_{\xi^{\prime}}$ for $\xi, \xi^{\prime} \in a_{q}$ or $\xi, \xi^{\prime} \in a_{r}$ are included in $n$. Consequently, again applying a) in a similar way for $q$ or $r$, already knowing that $k \in A_{\xi} \cap A_{\xi^{\prime}}$, we conclude that if i) holds for $k$, then $k$ does not belong to any $A_{\eta}$ for $\eta \in a_{p}-X$, which implies d).

A similar but simpler argument shows that the sets $D_{n}=\left\{p \in P: n_{p} \geq n\right\}$ are dense in $P$ for $n \in N$. The sets $E_{\xi}=\left\{p: \xi \in a_{p}\right\}$ are dense in $P$ for each $\xi \in \kappa$ because by the density of $D_{n}$ 's one can get $n_{p}$ large enough so that adding $\xi$ to $a_{p}$ does not violate a).

By Martin's axiom there is a filter $G \subseteq P$ which intersects all sets $E_{\xi}$ and $D_{n}$ for $n \in N$ and $\xi \in \kappa$. Then $\bigcup\left\{f_{p}: p \in G\right\}$ is a function that satisfies the lemma. Indeed, c) implies 1) and d) implies 2).

Proposition 6. Assume $M A_{\kappa}$ and suppose that $\mathcal{A}=\left\{A_{\xi}: \xi<\kappa\right\}$ is an almost disjoint family. For any $X \subseteq \kappa$ there is a decomposition $C\left(K_{\mathcal{A}}\right)=A \oplus B$ such that for suitable $\left\{n_{\xi}: \xi<\kappa\right\} \subseteq N$ we have $d_{\xi}^{n_{\xi}} \in A$ for $\xi \in X$ and $d_{\xi}^{n_{\xi}} \in B$ for $\eta \in \kappa-X$. In particular if both $X$ and $\kappa-X$ are uncountable, then both of the factors are non-separable.

Proof. Let $f$ be as in Lemma 5 for $X$. Define $r: K_{\mathcal{A}} \rightarrow K_{\mathcal{A}}$ in the following way: $r(n)=f(n)$ for $n \in N, r\left(x_{\xi}\right)=x_{\xi}$ for $\xi \in X, r\left(x_{\xi}\right)=\infty$ for $\xi \in \kappa-X$ and $r(\infty)=\infty$.

Let us check that $r$ is a retraction. It is clear that $r \circ r=r$, so we need to check that it is continuous. It is vacuously true at points of $N$. If $n_{i} \rightarrow x_{\xi}$ for $\xi \in X$, then $f\left(n_{i}\right) \rightarrow x_{\xi}=f\left(x_{\xi}\right)$ by 1$)$ of Lemma 5 and by the fact that $f$ is finite-to-one. If $n_{i} \rightarrow x_{\xi}$ for $\xi \in \kappa-X$, then $f\left(n_{i}\right) \rightarrow \infty=f\left(x_{\xi}\right)$ by 2) of Lemma 5 , by the part $f^{-1}\left[A_{\xi}\right] \subseteq^{*} A_{\xi}$ for $\xi \in X$ of 1$)$ of Lemma 5 and by the fact that $f$ is finite-to-one. Finally if $M \subseteq N$ has $\infty$ in its closure, then its image cannot be included in finitely many elements of the almost disjoint family by Lemma 5 , i.e. the image has $\infty$ in its closure as well, i.e., $f$ is continuous at $\infty$ as well.

Let $K=f[N] \cup\left\{x_{\xi}: \xi \in X\right\} \cup\{\infty\} \subseteq K_{\mathcal{A}}$ be the image of $r$. It is well known $(17, \S 21)$ that $C(K)$ is isomorphic to a subspace $A$ of $C\left(K_{\mathcal{A}}\right)$ defined by $\{f \circ r: f \in C(K)\}$ and that $P(f)=(f \mid K) \circ r=f \circ r$ is a projection witnessing the fact that $A$ is complemented in $C\left(K_{\mathcal{A}}\right)$. Note that $P\left(d_{\xi}^{n_{\xi}}\right)=d_{\xi}^{n_{\xi}}$ for some $n_{\xi} \in N$ if $\xi \in X$ by 1 ) of Lemma 5) and that $P\left(d_{\xi}^{n_{\xi}}\right)=0$ if $A_{\xi}-n_{\xi}$ is disjoint from $f[N]$ which can be acomplished by 2) of Lemma 5) for $\xi \in \kappa-X$.

\section{ACKNOWLEDGMENT}

We are thankful to Professor Joan Bagaria for organizing the visit at CRM.

\section{REFERENCES}

[1] D. Alspach, Y. Benyamini; Primariness of spaces of continuous functions on ordinals; Israel J. Math. vol 27, No 1, 1977, pp. 64 - 92. MR0440349 (55:13224)

[2] J. Diestel; Sequences and series in Banach spaces; Springer-Verlag 1984. MR0737004 (85i:46020) 
[3] E. van Douwen; The Integers and Topology; Handbook of Set-theoretic Topology; eds K. Kunen, J. Vaughan, North Holland 1984; pp. 111 - 167. MR0776622 (87f:54008)

[4] W. T. Gowers, B. Maurey; The unconditional basic sequence problem; Journal A. M. S. 6 (1993), pp. 851-874. MR1201238 (94k:46021)

[5] W. Johnson, J. Lindenstrauss; Some remarks on weakly compactly generated Banach spaces; Israel J. Math. 17, 1974, pp. 219 - 230. and Israel J. Math. 32 (1979), no. 4, pp. 382 - 383. MR 0417760 (54:5808) MR.0571092 (81g:46015)

[6] P. Koszmider; Banach spaces of large densities but few operators. Preprint.

[7] P. Koszmider; Banach spaces of continuous functions with few operators; Math. Annalen. 330, 2004, pp. 151 - 183. MR2091683

[8] K. Kunen; Set Theory. An Introduction to Independence Proofs; North Holland, 1980. MR:0597342 (82f:03001)

[9] Y. Lindenstrauss, A. Pełczyński; Contributions to the theory of the classical Banach spaces; J. Funct. Anal. 8, 1971, pp. 225 - 249. MR0291772 (45:863)

[10] N. Luzin; On subsets of the series of natural numbers; Izv. Akad. Nauk SSSR, Ser. Mat.., 11, pp. 403 - 411. MR0021576 (9:82c)

[11] A. Miller; private notes, 2003.

[12] A. Moltó; On a theorem of Sobczyk; Bull. Austral. Math. Soc. 43, 1991, 123 - 130. MR1086724 (92d:46043)

[13] S. Mrówka; Some set-theoretic constructions in topology; Fund. Math. vol. XCIV, 1977, pp. 83 - 92. MR0433388 (55:6364)

[14] A. Pełczyński; Projections in certain Banach spaces; Studia Math. vol. XIX, 1960, pp. 209 228. MR0126145 (23:A3441)

[15] A. Pełczyński, Z. Semadeni; Spaces of continuous functions (III) (Spaces $C(\Omega)$ for $\Omega$ without perfect subsets); Studia Math. 18, 1959, pp. 211 - 222. MR0107806 (21:6528)

[16] H. Rosenthal; On relatively disjoint families of measures with some applications to Banach space theory; Studia Math. 37, (1970), pp. 13-36. MR0270122 (42:5015)

[17] Z. Semadeni; Banach spaces of continuous functions; Państwowe Wydawnictwo Naukowe, 1971. MF0296671 (45:5730)

[18] S. Shelah; A Banach space with few operators; Israel J. Math. 30 (1978), pp. 181-191. MR 0508262 (80b:46033)

[19] S. Shelah, J. Steprans; A Banach space on which there are few operators; Proc. Amer. Math. Soc. 104 (1988), pp. 101-105. MR0958051 (90a:46047)

[20] H. Wark; A non-separable reflexive Banach space on which there are few operators. J. London Math. Soc. (2) 64 (2001), no. 3, pp. 675 - 689. MR1865556 (2003a:46031)

Departamento de Matemática, Universidade de São Paulo, Caixa Postal: 66281, São Paulo, Sp CEP: 05315-970, Brazil

E-mail address: piotr@ime.usp.br 